\title{
Floristic and ecological attributes of a Seasonal Semideciduous Atlantic Forest in a key area for conservation of the Zona da Mata region of Minas Gerais State, Brazil ${ }^{1}$
}

\author{
Maria José Reis da Rocha², Mônica A. Cupertino-Eisenlohrr,7, Lúcio S. Leoni ${ }^{4}$, \\ Aderbal Gomes da Silva ${ }^{5}$ and Mauro Eloi Nappo ${ }^{6}$
}

Received: 7.5.2016; accepted: 8.12.2016

\begin{abstract}
Floristic and ecological attributes of a Seasonal Semideciduous Atlantic Forest in a key area for conservation of the Zona da Mata region of Minas Gerais State, Brazil). We evaluated the floristic and ecological attributes of a seasonal semideciduous forest remnant (SSF) (500-1,050 m) located in a key area for biodiversity conservation. The importance of the fragment to the ecosystem conservation was revealed by its species richness (253), diversity (Shannon index $=$ 4.6; Simpson's complementary index $=0.98$ ) and evenness (Pielou index $=0.83$ ), which are considered high values for a SSF. Zoochory occurred in $77 \%$ of the species, indicating the existence of important biotic interactions that ensure the occurrence of gene flow. We recorded 14 endangered species, among which two are rare (Trigynaea oblongifolia and Trattinnickia ferruginea) and two are indicators of mature forests (Trattinnickia ferruginea and Virola bicuhyba). We confirmed the importance of key areas for biodiversity conservation and of the Atlantic SSF located at the 0-1,000 m altitudinal range. These areas deserve attention from conservationists due to the high species richness and high number of endemic and endangered species.
\end{abstract}

Keywords: Floristic Survey, Endangered Species, Hotspot, Species Distribution

RESUMO - (Atributos florísticos e ecológicos de uma Floresta Estacional Semidecidual Atlântica em área-chave para a conservação na Zona da Mata do Estado de Minas Gerais, Brasil). Avaliamos atributos florísticos e ecológicos de um remanescente de Floresta Estacional Semidecidual (FESD) (500-1.050 m) localizado em uma área-chave para conservação da biodiversidade. Evidenciamos a importância da conservação do fragmento devido à riqueza de espécies arbóreas (253), diversidade (Índice de Shannon=4,6; Índice complementar de Simpson =0,98) e equabilidade (Índice de Pielou =0,83), que são considerados valores altos para uma FESD. A síndrome zoocórica representou $77 \%$ das espécies, sugerindo a existência de interações bióticas importantes para a manutenção do fluxo gênico. Registramos 14 espécies ameaçadas de extinção, dentre as quais duas raras (Trigynaea oblongifolia e Trattinnickia ferruginea) e duas indicadoras de florestas maduras (Trattinnickia ferruginea e Virola bicuhyba). Confirmamos a importância de áreas-chave para a conservação da biodiversidade e da FESD Atlântica localizada em cotas altitudinais de 0-1.000 m, a qual merece atenção dos conservacionistas por apresentarem alta riqueza de espécies e elevado número de espécies endêmicas e ameaçadas.

Palavras-chave: Distribuição de Espécies, Espécies Ameaçadas, Hotspot, Levantamento Florístico

\section{Introduction}

Key areas for biodiversity conservation are sites of high value on a global scale (Giulietti et al. 2009).
Such key areas are geographic units large enough to maintain viable populations and can be identified by criteria based on species vulnerability and endemism (Langhammer et al. 2007, Giulietti et al. 2009). In

1. Parte da Dissertação de Mestrado do primeiro Autor

2. Universidade Federal de Minas Gerais, Instituto de Ciências Biológicas, Departamento de Botânica, Laboratório de Sistemática Vegetal. Av. Antônio Carlos, Pampulha, 30123-970 Belo Horizonte, Minas Gerais, Brasil

3. Universidade de Brasília, Instituto de Ciências Biológicas, Campus Darcy Ribeiro, Departamento de Botânica, 70919-970, Brasília, Distrito Federal, Brasil

4. Instituto de Ciências Naturais, Herbário Guido Pabst - GFJF, Santa Emília, 36800-000 Carangola, Minas Gerais, Brasil

5. Universidade Federal de São João Del-Rei, Campus Sete Lagoas, Departamento de Ciências Agrárias, Centro, 35701-970 Sete Lagoas, Minas Gerais, Brasil

6. Universidade de Brasília, Campus Darcy Ribeiro, Faculdade de Tecnologia, Departamento de Engenharia Florestal, 90910-900 Brasília, Distrito Federal, Brasil

7. Corresponding author: monicacupertino88@gmail.com 
Brazil, the analysis of the occupation of key areas for biodiversity conservation reveals a predominance of these areas in the Atlantic Forest (Giulietti et al. 2009).

The Atlantic Forest is one of the 35 biodiversity hotspots in the world (Zachos \& Habel 2011). More than $80 \%$ of its remnants have less than 50 ha; such remnants are damaged by border effect and by the distance between fragments (Ribeiro et al. 2009). These small fragments are also among the priorities for conservation of the Atlantic Forest and as such must be properly managed to keep the functional link among the vegetation mosaics, minimize border effect and improve connectivity between fragments (Ribeiro et al. 2009).

Connectivity between fragments aims to aid individual and gene flow among populations and subpopulations and to increase their chances of survival, thus enabling the maintenance of ecological and evolutionary processes on a large scale (Ayres et al. 2005). Management of connectivity between fragments has been made by means of ecological corridors. The Central Corridor of the Atlantic Forest, for instance, currently encompasses 11 areas of high priority for conservation of the region and possesses the highest vascular plant diversity in the world (Ayres et al. 2005).

The Parque Nacional do Caparaó, located between Minas Gerais and Espírito Santo States, Brazil, integrates the Central Corridor of the Atlantic Forest. Furthermore, that park, along with the Parque Estadual da Serra do Brigadeiro and their respective surrounding forest fragments, form a key area for biodiversity conservation (Giulietti et al. 2009). We aimed to study issues of floristic, ecological, and conservationist interest in a forest remnant between those two Protected Areas, by addressing the following questions: 1) What are the tree species composition and diversity in the area? 2) Which is the predominant seed dispersal syndrome in the area? 3) Are there endemic tree species in the studied fragment? 4) Which and how many tree species are threatened with extinction on a statewide (Red List of Plant Species Threatened with Extinction in Minas Gerais State; Biodiversitas 2008), nationwide (Martinelli \& Moraes 2013), and worldwide scale (Red List of Threatened Species of the International Union for Conservation of Nature-IUCN)? 5) Which and how many species are classified as rare, following Giulietti et al. (2009) and Martinelli \& Moraes (2013)? While answering these questions, we also aimed to address their implications on biodiversity conservation.

\section{Material and methods}

The study area consists in a Montane Seasonal Semideciduous Forest remnant in the Zona da Mata region of Minas Gerais State, Brazil, located in the surroundings of the Central Corridor of the Atlantic Forest, between Parque Nacional do Caparaó and Parque Estadual da Serra do Brigadeiro (figure 1). The region is considered key for the conservation of rare species (Giulietti et al. 2009).

The studied forest remnant is located within the private property Santa Rita Farm $\left(20^{\circ} 46^{\prime} \mathrm{S}, 42^{\circ} 02^{\prime} \mathrm{W}\right)$, at Faria Lemos municipality, on the watershed of Paraíba do Sul river. The fragment extends throughout an area of ca. 219 ha, with altitudes ranging between 500 and $1,050 \mathrm{~m}$. The history of occupation of the site reveals areas with different land uses: recovering areas, which were deforested in the 1960s and 1970s for coffee cultivation and wood exploitation; and predominantly well-preserved forest patches that did not undergo significant anthropic action.
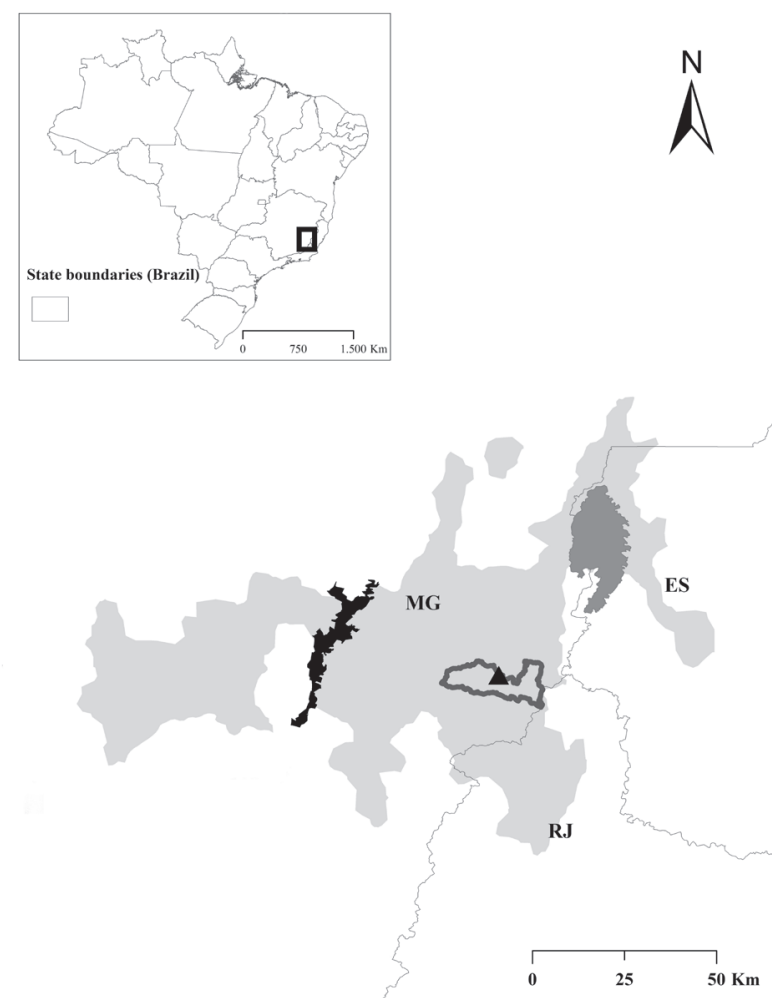

Figure 1. Geographic location of the Seasonal Semideciduous Atlantic Forest fragment at Santa Rita Farm, Faria Lemos municipality, Minas Gerais State, Brazil. Key areas for conservation of rare plants, $\square$ Parque Estadual da Serra do Brigadeiro, $\square$ Parque Nacional do Caparaó, $\mathbf{\Delta}$ S Santa Rita Farm, Faria Lemos, Minas Gerais State, Brazil. 
Koppen's climate in the region is Tropical Aw, characterized by high temperatures, with an annual mean of $24.5{ }^{\circ} \mathrm{C}$ (Peel et al. 2007). Mean annual precipitation is $1,200 \mathrm{~mm}$, with high values being registered in the rainy season, which takes place from October through April.

For vegetation sampling, we established three transects $20 \mathrm{~m}$ wide and $400 \mathrm{~m}$ long each, parallel and distant $200 \mathrm{~m}$ from one another. In each transect we established $11400-\mathrm{m}^{2}$ plots $(20 \times 20 \mathrm{~m})$, totaling 33 plots and 1.32 ha of sampling area. Plots were placed in order to be equidistant $20 \mathrm{~m}$ from one another along transects. Then, we sampled all tree individuals having circumference at $1.30 \mathrm{~m}$ above ground level equal to or higher than $15 \mathrm{~cm}$. Individuals with tillers below the 1.30-m mark above ground level had all their bifurcations included in the sampling whenever at least one of the tillers met the sampling criterion. Then, we measured the perimeter of each of the other bifurcations separately and calculated their respective basal areas; only then we calculated the sum of the basal area of tillers (Moro \& Martins 2011). Botanical material was identified by consultation to herbaria Guido Frederico João Pabst (GFJP) and Herbário do Jardim Botânico do Rio de Janeiro (RB), review of specialized literature, and by sending duplicates to specialists. Species were grouped in families following the classification system APG III (2009) and species' authors were checked in the online platforms Missouri Botanical Garden (http://www.mobot.org/) and Flora do Brasil (http://floradobrasil.jbrj.gov.br/). All collected botanical material was deposited in the GFJP herbarium, in Carangola city, Minas Gerais State.

Floristic diversity in the study area was estimated by species richness, Simpson's complementary index (1-D), and Shannon-Wiener (H') and Pielou's evenness $(\mathrm{J}$ ') indexes.

Species distribution was investigated based on two endemism criteria: (i) endemic to Brazil, and (ii) endemic to the Atlantic Forest. From this stage, we used only specimens identified at the species level in order to obtain higher data reliability in our analyses. To access such endemism criteria, we consulted databases Flora do Brasil (http://floradobrasil.jbrj. gov.br/) and SpeciesLink (http://splink.cria.org.br/), and the following literature: Catálogo das Árvores Nativas de Minas Gerais (Oliveira-Filho 2006), Plantas da Floresta Atlântica (Stehmann et al. 2009), and Árvores da Floresta Estacional Semidecidual Guia de Identificação de Espécies (Ramos et al. 2008). Ecological representativeness of species was evaluated by their dispersal syndrome, which is an important attribute to characterize environments and can be measured by the amount of plants that have zoochoric or the different abiotic syndromes (Campassi 2006). In that sense, we classified syndromes under categories zoochoric or abiotic based on diaspore morphology and on the review of literature on fruit and seed dispersal (e.g., van der Pijl 1982, Morellato et al. 2000, Catharino et al. 2006, Kinoshita et al. 2006, Campassi 2006, Yamamoto et al. 2007, Aquino \& Barbosa 2009, Prado-Júnior et al. 2012).

The degree of threat to the sampled species was evaluated by consultation to the IUCN Red List of Threatened Species (http://www.iucnredlist.org/), Livro Vermelho da Flora do Brasil (Martinelli \& Moraes 2013), and Lista Vermelha das Espécies da Flora Ameaçada de Extinção em Minas Gerais (Biodiversitas 2008). The rarity status of species was determined following Giulietti et al. (2009) and Martinelli \& Moraes (2013).

\section{Results and Discussion}

We identified 253 tree species belonging to 130 genera and 49 botanical families. The families that showed highest species richness were Fabaceae (26 species), Lauraceae (21), Myrtaceae (19), Rubiaceae (16), and Annonaceae (10) (table 1).

Fabaceae, Rubiaceae, and Myrtaceae are among the 10 most representative botanical families in the Brazilian flora (BFG 2015). These families are also among the richest ones in terms of species and endemism in the Atlantic Forest (Stehmann et al. 2009).

Fabaceae genera that show high richness in the Atlantic Forest were found in the study area, such as Inga Mill. and Machaerium Pers. (Stehmann et al. 2009). According to Oliveira-Filho \& Fontes (2000), the abovementioned genera are the most important ones in the Fabaceae that occur in seasonal forests, which is the case of the forest remnant we studied.

The most representative genera in the study area were Ocotea Aubl., with seven species; Eugenia L., Myrcia DC. and Licania Aubl., with five species, each; and Aspidosperma Mart., Cupania L., and Trichilia P. Browne, with four species, each. Ocotea is the most species-rich genus of Lauraceae in Brazil, while Eugenia and Myrcia are the most diverse Myrtaceae genera (BFG 2015) and are among the 20 most diverse genera in the Atlantic Forest (Stehmann et al. 2009).

Other less diverse genera found, yet that are represented among the richest ones in the Brazilian 
Table 1. List of angiosperm tree species found in a Seasonal Semideciduous Atlantic Forest remnant located at Santa Rita Farm, Faria Lemos municipality, Minas Gerais State, Brazil.

\begin{tabular}{|c|c|c|c|c|}
\hline Family/Species & $\begin{array}{c}\text { Collector, } \\
\text { Collector Number }\end{array}$ & $\begin{array}{l}\text { Endemic } \\
\text { to Brazil }\end{array}$ & $\begin{array}{l}\text { Endemic to the } \\
\text { Atlantic Forest }\end{array}$ & $\begin{array}{l}\text { Dispersal } \\
\text { Syndrome }\end{array}$ \\
\hline \multicolumn{5}{|l|}{ Achariaceae } \\
\hline Carpotroche brasiliensis (Raddi) A.Gray & Rocha, M.J.R., 1005 & Yes & No & Zoochory \\
\hline \multicolumn{5}{|l|}{ Anacardiaceae } \\
\hline Astronium fraxinifolium Schott & Rocha, M.J.R., 1006 & No & No & Abiotic \\
\hline Anacardiaceae 1 & Rocha, M.J.R., 1007 & No & No & - \\
\hline \multicolumn{5}{|l|}{ Annonaceae } \\
\hline Anaxagorea dolichocarpa Sprague \& Sandwith & Rocha, M.J.R., 1008 & No & No & Zoochory \\
\hline Annona cacans Warm. & Rocha, M.J.R., 1009 & No & Yes & Zoochory \\
\hline Guatteria campestris R.E. Fr. & Rocha, M.J.R., 1010 & Yes & Yes & Zoochory \\
\hline Guatteria pogonopus Mart. & Rocha, M.J.R., 1011 & Yes & Yes & Zoochory \\
\hline Guatteria sellowiana Schltdl. & Rocha, M.J.R., 1012 & Yes & No & Zoochory \\
\hline Oxandra nitida R.E. Fr. & Rocha, M.J.R., 1013 & Yes & Yes & Zoochory \\
\hline Trigynaea oblongifolia Schltdl. & Rocha, M.J.R., 1014 & Yes & Yes & Zoochory \\
\hline Xylopia brasiliensis Spreng. A.St.-Hil. & Rocha, M.J.R., 1015 & Yes & Yes & Zoochory \\
\hline Xylopia sericea A.St.-Hil. & Rocha, M.J.R., 1016 & No & No & Zoochory \\
\hline Xylopia sp. 1 & Rocha, M.J.R., 1017 & No & No & Zoochory \\
\hline \multicolumn{5}{|l|}{ Apocynaceae } \\
\hline Aspidosperma parvifolium A.DC. & Rocha, M.J.R., 1018 & No & No & Abiotic \\
\hline Aspidosperma polyneuron Müll.Arg. & Rocha, M.J.R., 1019 & No & No & Abiotic \\
\hline Aspidosperma sp. 1 & Rocha, M.J.R., 1020 & No & No & Abiotic \\
\hline Aspidosperma sp. 2 & Rocha, M.J.R., 1021 & No & No & Abiotic \\
\hline Himatanthus bracteatus (A.DC.) Woodson & Rocha, M.J.R., 1022 & Yes & Yes & Abiotic \\
\hline Tabernaemontana hystrix Steud. & Rocha, M.J.R., 1023 & Yes & Yes & Zoochory \\
\hline Apocynaceae 1 & Rocha, M.J.R., 1024 & No & No & - \\
\hline Apocynaceae 2 & Rocha, M.J.R., 1025 & No & No & - \\
\hline \multicolumn{5}{|l|}{ Aquifoliaceae } \\
\hline Ilex cerasifolia Reissek & Rocha, M.J.R., 1026 & Yes & Yes & Zoochory \\
\hline \multicolumn{5}{|l|}{ Araliaceae } \\
\hline $\begin{array}{l}\text { Schefflera morototoni (Aubl.) Maguire, } \\
\text { Steyerm. \& Frodin }\end{array}$ & Rocha, M.J.R., 1027 & No & No & Zoochory \\
\hline \multicolumn{5}{|l|}{ Arecaceae } \\
\hline Astrocaryum aculeatissimum (Schott) Burret & Rocha, M.J.R., 1028 & Yes & Yes & Zoochory \\
\hline Euterpe edulis Mart. & Rocha, M.J.R., 1029 & No & No & Zoochory \\
\hline Syagrus romanzoffiana (Cham.) Glassman & Rocha, M.J.R., 1030 & No & No & Zoochory \\
\hline \multicolumn{5}{|l|}{ Asteraceae } \\
\hline Piptocarpha macropoda (DC.) Baker & Rocha, M.J.R., 1031 & Yes & No & Abiotic \\
\hline Vernonanthura discolor (Spreng.) H. Rob. & Rocha, M.J.R., 1032 & No & No & Abiotic \\
\hline Asteraceae 1 & Rocha, M.J.R., 1033 & No & No & - \\
\hline \multicolumn{5}{|l|}{ Bignoniaceae } \\
\hline Jacaranda sp. 1 & Rocha, M.J.R., 1034 & No & No & Abiotic \\
\hline Sparattosperma leucanthum (Vell.) K.Schum. & Rocha, M.J.R., 1035 & No & No & Abiotic \\
\hline
\end{tabular}


Table 1 (continuation)

\begin{tabular}{|c|c|c|c|c|}
\hline Family/Species & $\begin{array}{c}\text { Collector, } \\
\text { Collector Number }\end{array}$ & $\begin{array}{l}\text { Endemic } \\
\text { to Brazil }\end{array}$ & $\begin{array}{l}\text { Endemic to the } \\
\text { Atlantic Forest }\end{array}$ & $\begin{array}{l}\text { Dispersal } \\
\text { Syndrome }\end{array}$ \\
\hline Tabebuia $\mathrm{sp} 1$ & Rocha, M.J.R., 1036 & No & No & Abiotic \\
\hline Zeyheria tuberculosa (Vell.) Bureau ex Verl. & Rocha, M.J.R., 1037 & No & No & Abiotic \\
\hline \multicolumn{5}{|l|}{ Burseraceae } \\
\hline Protium heptaphyllum (Aubl.) Marchand & Rocha, M.J.R., 1038 & No & No & Zoochory \\
\hline Protium tenuifolium (Engl.) Engl. & Rocha, M.J.R., 1039 & No & No & Zoochory \\
\hline Trattinnickia ferruginea Kuhlm. & Rocha, M.J.R., 1040 & Yes & Yes & Zoochory \\
\hline \multicolumn{5}{|l|}{ Caricaceae } \\
\hline Jacaratia spinosa (Aubl.) A.DC. & Rocha, M.J.R., 1041 & No & No & Zoochory \\
\hline \multicolumn{5}{|l|}{ Celastraceae } \\
\hline Cheiloclinium anomalum Miers & Rocha, M.J.R., 1042 & No & No & Zoochory \\
\hline Maytenus sp1 & Rocha, M.J.R., 1043 & No & No & Zoochory \\
\hline Tontelea corcovadensis A.C. Sm & Rocha, M.J.R., 1044 & Yes & Yes & Zoochory \\
\hline Celastraceae 1 & Rocha, M.J.R., 1045 & No & No & - \\
\hline \multicolumn{5}{|l|}{ Chrysobalanaceae } \\
\hline Hirtella hebeclada Moric. ex DC. & Rocha, M.J.R., 1046 & Yes & No & Zoochory \\
\hline Hirtella sp. 1 & Rocha, M.J.R., 1047 & No & No & Zoochory \\
\hline Licania cf. blackii Prance & Rocha, M.J.R., 1048 & No & No & - \\
\hline Licania kunthiana Hook.f. & Rocha, M.J.R., 1049 & Yes & No & Zoochory \\
\hline Licania sp. 1 & Rocha, M.J.R., 1050 & No & No & Zoochory \\
\hline Licania sp. 2 & Rocha, M.J.R., 1051 & No & No & Zoochory \\
\hline Licania sp. 3 & Rocha, M.J.R., 1052 & No & No & Zoochory \\
\hline Chrysobalanaceae 1 & Rocha, M.J.R., 1053 & No & No & - \\
\hline \multicolumn{5}{|l|}{ Clusiaceae } \\
\hline Clusia sp. 1 & Rocha, M.J.R., 1054 & No & No & - \\
\hline $\begin{array}{l}\text { Garcinia gardneriana (Planch. \& Triana) } \\
\text { Zappi }\end{array}$ & Rocha, M.J.R., 1055 & No & No & Zoochory \\
\hline Tovomita cf. riedeliana Engl. & Rocha, M.J.R., 1056 & No & No & Zoochory \\
\hline Tovomita sp. 1 & Rocha, M.J.R., 1057 & No & No & Zoochory \\
\hline Tovomitopsis sp. 1 & Rocha, M.J.R., 1058 & Yes & Yes & Zoochory \\
\hline Clusiaceae 1 & Rocha, M.J.R., 1059 & No & No & Zoochory \\
\hline \multicolumn{5}{|l|}{ Combretaceae } \\
\hline Buchenavia sp. 1 & Rocha, M.J.R., 1060 & No & No & Zoochory \\
\hline \multicolumn{5}{|l|}{ Elaeocarpaceae } \\
\hline Sloanea guianensis (Aubl.) Benth. & Rocha, M.J.R., 1061 & No & No & Zoochory \\
\hline Sloanea hirsuta (Schott) Planch. ex Benth. & Rocha, M.J.R., 1062 & Yes & Yes & Zoochory \\
\hline \multicolumn{5}{|l|}{ Erythroxylaceae } \\
\hline Erythroxylum citrifolium A.St.-Hil. & Rocha, M.J.R., 1063 & No & No & Zoochory \\
\hline Erythroxylum pulchrum A.St.-Hil. & Rocha, M.J.R., 1064 & Yes & Yes & Zoochory \\
\hline \multicolumn{5}{|l|}{ Euphorbiaceae } \\
\hline Joannesia princeps Vell. & Rocha, M.J.R., 1065 & Yes & No & Zoochory \\
\hline Mabea fistulifera Mart. & Rocha, M.J.R., 1066 & No & No & Zoochory \\
\hline
\end{tabular}


Table 1 (continuation)

\begin{tabular}{|c|c|c|c|c|}
\hline Family/Species & $\begin{array}{c}\text { Collector, } \\
\text { Collector Number }\end{array}$ & $\begin{array}{l}\text { Endemic } \\
\text { to Brazil }\end{array}$ & $\begin{array}{l}\text { Endemic to the } \\
\text { Atlantic Forest }\end{array}$ & $\begin{array}{l}\text { Dispersal } \\
\text { Syndrome }\end{array}$ \\
\hline Sapium sp. 1 & Rocha, M.J.R., 1067 & No & No & Zoochory \\
\hline Sebastiania sp. & Rocha, M.J.R., 1068 & No & No & Abiotic \\
\hline Senefeldera verticillata (Vell.) Croizat & Rocha, M.J.R., 1069 & Yes & Yes & Abiotic \\
\hline Euphorbiaceae 1 & Rocha, M.J.R., 1070 & No & No & - \\
\hline \multicolumn{5}{|l|}{ Fabaceae } \\
\hline Anadenanthera colubrina (Vell.) Brenan & Rocha, M.J.R., 1071 & No & No & Abiotic \\
\hline Anadenanthera peregrina (L.) Speg & Rocha, M.J.R., 1072 & No & No & Abiotic \\
\hline Apuleia leiocarpa (Vogel) J.F. Macbr. & Rocha, M.J.R., 1073 & No & No & Abiotic \\
\hline Bauhinia sp. 1 & Rocha, M.J.R., 1074 & No & No & Abiotic \\
\hline Cassia ferruginea (Schrad.) DC. & Rocha, M.J.R., 1075 & No & No & Abiotic \\
\hline Copaifera trapezifolia Hayne & Rocha, M.J.R., 1076 & No & Yes & Zoochory \\
\hline Dalbergia nigra (Vell.) Benth. & Rocha, M.J.R., 1077 & Yes & Yes & Abiotic \\
\hline Inga capitata Desv. & Rocha, M.J.R., 1078 & No & No & Zoochory \\
\hline Inga striata Benth. & Rocha, M.J.R., 1079 & No & No & Zoochory \\
\hline Inga vera Willd. & Rocha, M.J.R., 1080 & No & No & Zoochory \\
\hline Machaerium nyctitans (Vell.) Benth. & Rocha, M.J.R., 1081 & No & No & Abiotic \\
\hline Machaerium sp. 1 & Rocha, M.J.R., 1082 & No & No & Abiotic \\
\hline Melanoxylon brauna Schott & Rocha, M.J.R., 1083 & Yes & No & Abiotic \\
\hline Myrocarpus frondosus Allemao & Rocha, M.J.R., 1084 & No & Yes & Abiotic \\
\hline Myroxylon peruiferum L.f. & Rocha, M.J.R., 1085 & No & No & Abiotic \\
\hline Piptadenia gonoacantha Mart. J.F. Macbr. & Rocha, M.J.R., 1086 & No & Yes & Abiotic \\
\hline Platypodium elegans Vogel & Rocha, M.J.R., 1087 & No & No & Abiotic \\
\hline $\begin{array}{l}\text { Pseudopiptadenia contorta DC.) G.P. Lewis } \\
\text { \& M.P. Lima }\end{array}$ & Rocha, M.J.R., 1088 & Yes & No & Abiotic \\
\hline Swartzia sp.1 & Rocha, M.J.R., 1089 & No & No & - \\
\hline Vataireopsis araroba (Aguiar) Ducke & Rocha, M.J.R., 1090 & Yes & Yes & Abiotic \\
\hline Fabaceae 1 & Rocha, M.J.R., 1091 & No & No & - \\
\hline Fabaceae 2 & Rocha, M.J.R., 1092 & No & No & - \\
\hline Fabaceae 3 & Rocha, M.J.R., 1093 & No & No & - \\
\hline Fabaceae 4 & Rocha, M.J.R., 1094 & No & No & - \\
\hline Fabaceae 5 & Rocha, M.J.R., 1095 & No & No & - \\
\hline Fabaceae 6 & Rocha, M.J.R., 1096 & No & No & - \\
\hline \multicolumn{5}{|l|}{ Lacistemataceae } \\
\hline Lacistema pubescens Mart. & Rocha, M.J.R., 1097 & Yes & No & Zoochory \\
\hline \multicolumn{5}{|l|}{ Lamiaceae } \\
\hline Aegiphila integrifolia (Jacq.) B.D.Jacks. & Rocha, M.J.R., 1098 & No & No & Zoochory \\
\hline Vitex megapotamica (Spreng.) Moldenke & Rocha, M.J.R., 1099 & No & No & Zoochory \\
\hline \multicolumn{5}{|l|}{ Lauraceae } \\
\hline Aiouea saligna Meisn. & Rocha, M.J.R., 1100 & Yes & No & Zoochory \\
\hline Aiouea sp. 1 & Rocha, M.J.R., 1101 & No & No & Zoochory \\
\hline
\end{tabular}


Table 1 (continuation)

\begin{tabular}{|c|c|c|c|c|}
\hline Family/Species & $\begin{array}{c}\text { Collector, } \\
\text { Collector Number }\end{array}$ & $\begin{array}{l}\text { Endemic } \\
\text { to Brazil }\end{array}$ & $\begin{array}{l}\text { Endemic to the } \\
\text { Atlantic Forest }\end{array}$ & $\begin{array}{r}\text { Dispersal } \\
\text { Syndrome }\end{array}$ \\
\hline Aniba firmula (Nees \& Mart.) Mez & Rocha, M.J.R., 1102 & Yes & No & Zoochory \\
\hline Aniba sp. 1 & Rocha, M.J.R., 1103 & No & No & Zoochory \\
\hline $\begin{array}{l}\text { Beilschmiedia emarginata (Meisn.) } \\
\text { Kosterm. }\end{array}$ & Rocha, M.J.R., 1104 & Yes & Yes & Zoochory \\
\hline Licaria armeniaca (Nees) Kosterm. & Rocha, M.J.R., 1105 & No & No & Zoochory \\
\hline Nectandra cuspidata Nees \& Mart. & Rocha, M.J.R., 1106 & No & No & Zoochory \\
\hline Nectandra lanceolata Nees \& Mart. & Rocha, M.J.R., 1107 & Yes & No & Zoochory \\
\hline Ocotea citrosmoides (Nees) Mez & Rocha, M.J.R., 1108 & Yes & Yes & Zoochory \\
\hline Ocotea divaricata $(\mathrm{Nees}) \mathrm{Mez}$ & Rocha, M.J.R., 1109 & Yes & Yes & Zoochory \\
\hline Ocotea indecora $(\mathrm{Schott}) \mathrm{Mez}$ & Rocha, M.J.R., 1110 & Yes & Yes & Zoochory \\
\hline Ocotea sp. 1 & Rocha, M.J.R., 1111 & No & No & Zoochory \\
\hline Ocotea sp. 2 & Rocha, M.J.R., 1112 & No & No & Zoochory \\
\hline Ocotea sp. 3 & Rocha, M.J.R., 1113 & No & No & Zoochory \\
\hline Ocotea spixiana (Nees) Mez & Rocha, M.J.R., 1114 & Yes & No & Zoochory \\
\hline Lauraceae 1 & Rocha, M.J.R., 1115 & No & No & - \\
\hline Lauraceae 2 & Rocha, M.J.R., 1116 & No & No & - \\
\hline Lauraceae 3 & Rocha, M.J.R., 1117 & No & No & - \\
\hline Lauraceae 4 & Rocha, M.J.R., 1118 & No & No & - \\
\hline Lauraceae 5 & Rocha, M.J.R., 1119 & No & No & - \\
\hline Lauraceae 6 & Rocha, M.J.R., 1120 & No & No & - \\
\hline \multicolumn{5}{|l|}{ Lecythidaceae } \\
\hline Cariniana legalis (Mart.) Kuntze & Rocha, M.J.R., 1121 & Yes & Yes & Abiotic \\
\hline Lecythis sp. 1 & Rocha, M.J.R., 1122 & No & No & - \\
\hline \multicolumn{5}{|l|}{ Malpighiaceae } \\
\hline Byrsonima sp. 1 & Rocha, M.J.R., 1123 & No & No & Zoochory \\
\hline \multicolumn{5}{|l|}{ Malvaceae } \\
\hline Ceiba speciosa (A.St.-Hil.) Ravenna & Rocha, M.J.R., 1124 & No & No & Abiotic \\
\hline Eriotheca sp. 1 & Rocha, M.J.R., 1125 & No & No & - \\
\hline Eriotheca sp. 2 & Rocha, M.J.R., 1126 & No & No & - \\
\hline Luehea divaricata Mart. & Rocha, M.J.R., 1127 & No & No & Abiotic \\
\hline $\begin{array}{l}\text { Pseudobombax grandiflorum (Cav.) A. } \\
\text { Robyns }\end{array}$ & Rocha, M.J.R., 1128 & Yes & Yes & Abiotic \\
\hline Spirotheca sp. 1 & Rocha, M.J.R., 1129 & Yes & Yes & - \\
\hline Sterculia apetala (Jacq.) H. Karst. & Rocha, M.J.R., 1130 & No & No & Abiotic \\
\hline \multicolumn{5}{|l|}{ Melastomataceae } \\
\hline Miconia altissima Cogn. & Rocha, M.J.R., 1131 & Yes & Yes & Zoochory \\
\hline Miconia lepidota Schrank \& Mart. ex DC & Rocha, M.J.R., 1132 & No & No & Zoochory \\
\hline Tibouchina estrellensis (Raddi) Cogn. & Rocha, M.J.R., 1133 & Yes & Yes & Abiotic \\
\hline \multicolumn{5}{|l|}{ Meliaceae } \\
\hline Cedrela fissilis Vell. & Rocha, M.J.R., 1134 & No & No & Abiotic \\
\hline Guarea kunthiana A. Juss. & Rocha, M.J.R., 1135 & No & No & Zoochory \\
\hline
\end{tabular}


Table 1 (continuation)

\begin{tabular}{|c|c|c|c|c|}
\hline Family/Species & $\begin{array}{c}\text { Collector, } \\
\text { Collector Number }\end{array}$ & $\begin{array}{l}\text { Endemic } \\
\text { to Brazil }\end{array}$ & $\begin{array}{l}\text { Endemic to the } \\
\text { Atlantic Forest }\end{array}$ & $\begin{array}{l}\text { Dispersal } \\
\text { Syndrome }\end{array}$ \\
\hline Trichilia elegans A. Juss. & Rocha, M.J.R., 1136 & Yes & No & Zoochory \\
\hline Trichilia pallida $\mathrm{Sw}$. & Rocha, M.J.R., 1137 & No & No & Zoochory \\
\hline Trichilia $\mathrm{sp} .1$ & Rocha, M.J.R., 1138 & No & No & Zoochory \\
\hline Trichilia sp. 2 & Rocha, M.J.R., 1139 & No & No & Zoochory \\
\hline Meliaceae 1 & Rocha, M.J.R., 1140 & No & No & - \\
\hline Meliaceae 2 & Rocha, M.J.R., 1141 & No & No & - \\
\hline \multicolumn{5}{|l|}{ Monimiaceae } \\
\hline Mollinedia acutissima Perkins & Rocha, M.J.R., 1142 & Yes & Yes & Zoochory \\
\hline Mollinedia widgrenii A. DC. & Rocha, M.J.R., 1143 & Yes & No & Zoochory \\
\hline \multicolumn{5}{|l|}{ Moraceae } \\
\hline Brosimum glaziovii Taub. & Rocha, M.J.R., 1144 & Yes & Yes & Zoochory \\
\hline $\begin{array}{l}\text { Brosimum guianense (Aubl.) Huber ex } \\
\text { Ducke }\end{array}$ & Rocha, M.J.R., 1145 & No & No & Zoochory \\
\hline $\begin{array}{l}\text { Clarisia ilicifolia (Spreng.) Lanj. \& } \\
\text { Rossbach }\end{array}$ & Rocha, M.J.R., 1146 & No & No & Zoochory \\
\hline Ficus adhatodifolia Schott & Rocha, M.J.R., 1147 & No & No & Zoochory \\
\hline Ficus mariae C.C.Berg, Emygdio \& Carauta & Rocha, M.J.R., 1148 & No & Yes & Zoochory \\
\hline Ficus trigona L.f. & Rocha, M.J.R., 1149 & No & No & Zoochory \\
\hline $\begin{array}{l}\text { Sorocea bonplandii (Baill.) W.C.Burger, } \\
\text { Lanj. \& de Boer }\end{array}$ & Rocha, M.J.R., 1150 & No & No & Zoochory \\
\hline \multicolumn{5}{|l|}{ Myristicaceae } \\
\hline Virola bicuhyba (Schott) Warb. & Rocha, M.J.R., 1151 & Yes & Yes & Zoochory \\
\hline Virola gardneri (A.DC.) Warb. & Rocha, M.J.R., 1152 & Yes & Yes & Zoochory \\
\hline \multicolumn{5}{|l|}{ Myrtaceae } \\
\hline Campomanesia sp. 1 & Rocha, M.J.R., 1153 & No & No & Zoochory \\
\hline Eugenia cf. florida DC. & Rocha, M.J.R., 1154 & No & No & Zoochory \\
\hline Eugenia cf. magnifica Spring ex Mart. & Rocha, M.J.R., 1155 & No & No & Zoochory \\
\hline Eugenia sp. 1 & Rocha, M.J.R., 1156 & No & No & Zoochory \\
\hline Eugenia sp. 2 & Rocha, M.J.R., 1157 & No & No & Zoochory \\
\hline Eugenia sp. 3 & Rocha, M.J.R., 1158 & No & No & Zoochory \\
\hline Marlierea excoriata Mart. & Rocha, M.J.R., 1159 & Yes & Yes & Zoochory \\
\hline Myrcia guianensis (Aubl.) DC. & Rocha, M.J.R., 1160 & No & No & Zoochory \\
\hline Myrcia insigniflora M.F. Santos & Rocha, M.J.R., 1161 & Yes & Yes & Zoochory \\
\hline Myrcia sp. 1 & Rocha, M.J.R., 1162 & No & No & Zoochory \\
\hline Myrcia splendens (Sw.) DC. & Rocha, M.J.R., 1163 & Yes & No & Zoochory \\
\hline Myrcia tenuivenosa Kiaersk. & Rocha, M.J.R., 1164 & Yes & Yes & Zoochory \\
\hline $\begin{array}{l}\text { Myrciaria floribunda (H.West ex Willd.) } \\
\text { O. Berg }\end{array}$ & Rocha, M.J.R., 1165 & No & No & Zoochory \\
\hline Psidium guajava $\mathrm{L}$. & Rocha, M.J.R., 1166 & No & No & Zoochory \\
\hline Myrtaceae 1 & Rocha, M.J.R., 1167 & No & No & - \\
\hline Myrtaceae 2 & Rocha, M.J.R., 1168 & No & No & - \\
\hline
\end{tabular}


Table 1 (continuation)

\begin{tabular}{|c|c|c|c|c|}
\hline Family/Species & $\begin{array}{c}\text { Collector, } \\
\text { Collector Number }\end{array}$ & $\begin{array}{l}\text { Endemic } \\
\text { to Brazil }\end{array}$ & $\begin{array}{l}\text { Endemic to the } \\
\text { Atlantic Forest }\end{array}$ & $\begin{array}{l}\text { Dispersal } \\
\text { Syndrome }\end{array}$ \\
\hline Myrtaceae 3 & Rocha, M.J.R., 1169 & No & No & - \\
\hline Myrtaceae 4 & Rocha, M.J.R., 1170 & No & No & - \\
\hline Myrtaceae 5 & Rocha, M.J.R., 1171 & No & No & - \\
\hline \multicolumn{5}{|l|}{ Nyctaginaceae } \\
\hline Guapira opposita (Vell.) Reitz & Rocha, M.J.R., 1172 & No & No & Zoochory \\
\hline \multicolumn{5}{|l|}{ Olacaceae } \\
\hline Heisteria silvianii Schwacke & Rocha, M.J.R., 1173 & Yes & No & Zoochory \\
\hline Tetrastylidium grandifolium (Baill.) Sleumer & Rocha, M.J.R., 1174 & Yes & Yes & Zoochory \\
\hline \multicolumn{5}{|l|}{ Primulaceae } \\
\hline Myrsine umbellata Mart. & Rocha, M.J.R., 1175 & No & No & Zoochory \\
\hline \multicolumn{5}{|l|}{ Phyllanthaceae } \\
\hline Hieronyma alchorneoides Allemão & Rocha, M.J.R., 1176 & No & No & Zoochory \\
\hline \multicolumn{5}{|l|}{ Picramniaceae } \\
\hline Picramnia glazioviana Engl. & Rocha, M.J.R., 1177 & No & No & Zoochory \\
\hline \multicolumn{5}{|l|}{ Proteaceae } \\
\hline Roupala sp. 1 & Rocha, M.J.R., 1178 & No & No & Abiotic \\
\hline \multicolumn{5}{|l|}{ Rhamnaceae } \\
\hline Rhamnidium sp. 1 & Rocha, M.J.R., 1179 & No & No & Zoochory \\
\hline \multicolumn{5}{|l|}{ Rosaceae } \\
\hline Prunus myrtifolia (L.) Urb. & Rocha, M.J.R., 1180 & No & No & Zoochory \\
\hline \multicolumn{5}{|l|}{ Rubiaceae } \\
\hline Amaioua guianensis Aubl. & Rocha, M.J.R., 1181 & No & No & Zoochory \\
\hline Bathysa australis (A.St.-Hil.) K. Schum. & Rocha, M.J.R., 1182 & No & No & Abiotic \\
\hline Coussarea congestiflora Müll. Arg. & Rocha, M.J.R., 1183 & No & No & Zoochory \\
\hline Ixora brevifolia Benth. & Rocha, M.J.R., 1184 & - & No & Zoochory \\
\hline Posoqueria acutifolia Mart. & Rocha, M.J.R., 1185 & Yes & No & Zoochory \\
\hline Psychotria hastisepala Müll. Arg. & Rocha, M.J.R., 1186 & Yes & No & Zoochory \\
\hline Psychotria sp. 1 & Rocha, M.J.R., 1187 & No & No & Zoochory \\
\hline Psychotria vellosiana Benth. & Rocha, M.J.R., 1188 & No & No & Zoochory \\
\hline Randia armata (Sw.) DC. & Rocha, M.J.R., 1189 & No & No & Zoochory \\
\hline Randia sp. 1 & Rocha, M.J.R., 1190 & No & No & Zoochory \\
\hline $\begin{array}{l}\text { Rudgea jasminoides subsp. corniculata } \\
\text { (Benth.) Zappi }\end{array}$ & Rocha, M.J.R., 1191 & No & No & Zoochory \\
\hline Simira sampaioana (Standl.) Steyerm. & Rocha, M.J.R., 1192 & Yes & No & Abiotic \\
\hline $\begin{array}{l}\text { Tocoyena sellowiana (Cham. \& } \\
\text { Schltdl.) K.Schum. }\end{array}$ & Rocha, M.J.R., 1193 & Yes & No & Zoochory \\
\hline Rubiaceae 1 & Rocha, M.J.R., 1194 & No & No & - \\
\hline Rubiaceae 2 & Rocha, M.J.R., 1195 & No & No & - \\
\hline Rubiaceae 3 & Rocha, M.J.R., 1196 & No & No & - \\
\hline \multicolumn{5}{|l|}{ Rutaceae } \\
\hline Esenbeckia sp. 1 & Rocha, M.J.R., 1197 & No & No & - \\
\hline
\end{tabular}


Table 1 (continuation)

\begin{tabular}{|c|c|c|c|c|}
\hline Family/Species & $\begin{array}{c}\text { Collector, } \\
\text { Collector Number }\end{array}$ & $\begin{array}{l}\text { Endemic } \\
\text { to Brazil }\end{array}$ & $\begin{array}{l}\text { Endemic to the } \\
\text { Atlantic Forest }\end{array}$ & $\begin{array}{l}\text { Dispersal } \\
\text { Syndrome }\end{array}$ \\
\hline Zanthoxylum rhoifolium Lam. & Rocha, M.J.R., 1198 & No & No & Zoochory \\
\hline Zanthoxylum sp. 1 & Rocha, M.J.R., 1199 & No & No & Zoochory \\
\hline Rutaceae 1 & Rocha, M.J.R., 1200 & No & No & \\
\hline \multicolumn{5}{|l|}{ Salicaceae } \\
\hline Casearia decandra Jacq. & Rocha, M.J.R., 1201 & Yes & No & Zoochory \\
\hline Casearia sp. 1 & Rocha, M.J.R., 1202 & No & No & Zoochory \\
\hline Casearia sylvestris $\mathrm{Sw}$. & Rocha, M.J.R., 1203 & No & No & Zoochory \\
\hline Macrothumia kuhlmannii (Sleumer) Alford & Rocha, M.J.R., 1204 & Yes & Yes & Zoochory \\
\hline Xylosma prockia (Turcz.) Turcz. & Rocha, M.J.R., 1205 & No & No & Zoochory \\
\hline \multicolumn{5}{|l|}{ Sapindaceae } \\
\hline Allophylus sp. 1 & Rocha, M.J.R., 1206 & No & No & Zoochory \\
\hline Cupania ludowigii Somner \& Ferrucci & Rocha, M.J.R., 1207 & Yes & Yes & Zoochory \\
\hline Cupania sp. 1 & Rocha, M.J.R., 1208 & No & No & Zoochory \\
\hline Cupania sp. 2 & Rocha, M.J.R., 1209 & No & No & Zoochory \\
\hline Cupania vernalis Cambess. & Rocha, M.J.R., 1210 & No & No & Zoochory \\
\hline Matayba elaeagnoides Radlk. & Rocha, M.J.R., 1211 & No & No & Zoochory \\
\hline Sapindaceae 1 & Rocha, M.J.R., 1212 & No & No & - \\
\hline Sapindaceae 2 & Rocha, M.J.R., 1213 & No & No & - \\
\hline Sapindaceae 3 & Rocha, M.J.R., 1214 & No & No & - \\
\hline \multicolumn{5}{|l|}{ Sapotaceae } \\
\hline Chrysophyllum sp. 1 & Rocha, M.J.R., 1215 & No & No & Zoochory \\
\hline Micropholis gardneriana (A.DC.) Pierre & Rocha, M.J.R., 1216 & No & No & Zoochory \\
\hline Pouteria torta (Mart.) Radlk. & Rocha, M.J.R., 1217 & No & No & Zoochory \\
\hline \multicolumn{5}{|l|}{ Siparunaceae } \\
\hline Siparuna guianensis Aubl. & Rocha, M.J.R., 1218 & No & No & Zoochory \\
\hline Siparuna sp. 1 & Rocha, M.J.R., 1219 & No & No & Zoochory \\
\hline \multicolumn{5}{|l|}{ Solanaceae } \\
\hline Solanum intermedium Sendtn. & Rocha, M.J.R., 1220 & Yes & Yes & Zoochory \\
\hline Solanum sp. 1 & Rocha, M.J.R., 1221 & No & No & Zoochory \\
\hline \multicolumn{5}{|l|}{ Symplocaceae } \\
\hline Symplocos estrellensis Casar. & Rocha, M.J.R., 1222 & Yes & Yes & Zoochory \\
\hline Symplocos sp. 1 & Rocha, M.J.R., 1223 & No & No & - \\
\hline \multicolumn{5}{|l|}{ Urticaceae } \\
\hline Cecropia glaziovii Snethl. & Rocha, M.J.R., 1224 & Yes & Yes & Zoochory \\
\hline Cecropia hololeuca Miq. & Rocha, M.J.R., 1225 & Yes & Yes & Zoochory \\
\hline Coussapoa curranii S.F. Blake & Rocha, M.J.R., 1226 & Yes & No & Zoochory \\
\hline Pourouma guianensis Aubl & Rocha, M.J.R., 1227 & No & No & Zoochory \\
\hline \multicolumn{5}{|l|}{ Vochysiaceae } \\
\hline Qualea gestasiana A.St.-Hil. & Rocha, M.J.R., 1228 & Yes & Yes & Abiotic \\
\hline Vochysia sp. 1 & Rocha, M.J.R., 1229 & No & No & Abiotic \\
\hline Morphospecies 1 & Rocha, M.J.R., 1230 & & & \\
\hline
\end{tabular}


Table 1 (continuation)

\begin{tabular}{|c|c|c|c|c|}
\hline Family/Species & $\begin{array}{c}\text { Collector, } \\
\text { Collector Number }\end{array}$ & $\begin{array}{l}\text { Endemic } \\
\text { to Brazil }\end{array}$ & $\begin{array}{l}\text { Endemic to the } \\
\text { Atlantic Forest }\end{array}$ & $\begin{array}{l}\text { Dispersal } \\
\text { Syndrome }\end{array}$ \\
\hline Morphospecies 2 & Rocha, M.J.R., 1231 & & & \\
\hline Morphospecies 3 & Rocha, M.J.R., 1232 & & & \\
\hline Morphospecies 4 & Rocha, M.J.R., 1233 & & & \\
\hline Morphospecies 5 & Rocha, M.J.R., 1234 & & & \\
\hline Morphospecies 6 & Rocha, M.J.R., 1235 & & & \\
\hline Morphospecies 7 & Rocha, M.J.R., 1236 & & & \\
\hline Morphospecies 8 & Rocha, M.J.R., 1237 & & & \\
\hline Morphospecies 10 & Rocha, M.J.R., 1238 & & & \\
\hline Morphospecies 11 & Rocha, M.J.R., 1239 & & & \\
\hline Morphospecies 12 & Rocha, M.J.R., 1240 & & & \\
\hline Morphospecies 13 & Rocha, M.J.R., 1241 & & & \\
\hline Morphospecies 14 & Rocha, M.J.R., 1242 & & & \\
\hline Morphospecies 15 & Rocha, M.J.R., 1243 & & & \\
\hline Morphospecies 17 & Rocha, M.J.R., 1244 & & & \\
\hline Morphospecies 18 & Rocha, M.J.R., 1245 & & & \\
\hline Morphospecies 19 & Rocha, M.J.R., 1246 & & & \\
\hline Morphospecies 20 & Rocha, M.J.R., 1247 & & & \\
\hline Morphospecies 21 & Rocha, M.J.R., 1248 & & & \\
\hline Morphospecies 22 & Rocha, M.J.R., 1249 & & & \\
\hline Morphospecies 23 & Rocha, M.J.R., 1250 & & & \\
\hline Morphospecies 24 & Rocha, M.J.R., 1251 & & & \\
\hline Morphospecies 25 & Rocha, M.J.R., 1252 & & & \\
\hline Morphospecies 26 & Rocha, M.J.R., 1253 & & & \\
\hline Morphospecies 28 & Rocha, M.J.R., 1254 & & & \\
\hline Morphospecies 29 & Rocha, M.J.R., 1255 & & & \\
\hline Morphospecies 30 & Rocha, M.J.R., 1256 & & & \\
\hline Morphospecies 31 & Rocha, M.J.R., 1257 & & & \\
\hline
\end{tabular}

flora, were: Miconia Ruiz \& Pav., Solanum L., Psychotria L., and Tibouchina Aubl. Solanum, Miconia, and Tibouchina are also among the 20 most diverse genera in the Atlantic Forest (Stehmann et al. 2009).

Species diversity in the study area was 4.6 according to Shannon-Wiener's index (H') and 0.98 according to Simpson's complementary index (1-D). Pielou's evenness (J') was 0.83 . Meira-Neto \& Martins (2000) established maximum and minimum values for Shannon ( $\mathrm{H}^{\prime}$ between 3.2 and 4.2) and evenness ( $\mathrm{J}$ ' between 0.73 and 0.88 ) indexes to the Zona da Mata region of Minas Gerais State. Our data thus yielded results above those marks. In comparison with studies performed in other seasonal semideciduous forest remnants that used similar sampling methods (e.g., Silva et al. 2004, Marangon et al. 2007, Leite \& Rodrigues 2008, Dias-Neto et al. 2009, Braga et al. 2011, Santos et al. 2013, Coelho et al. 2016), the values of richness, diversity, and evenness found at Santa Rita Farm were also higher. The high values of those indexes found in our study provide evidence of the ecological importance of the studied fragment, which is located between two important Protected Areas (PAs) of the Atlantic Forest, namely the Parque Estadual da Serra do Brigadeiro and the Parque Nacional do Caparaó. In that sense, should an ecological corridor be implemented between those PAs, the remnant might well represent a strategic area for the maintenance of gene flow in the region. 
A total 140 specimens were identified at the species level. Regarding their distribution in Brazil, 77 species (55\%) are not endemic and $62(44 \%)$ are endemic to the country. This latter value reflects the pattern overall found in the Brazilian flora, which has a high degree of endemism of seed plants - 57.4\% of all phanerogams occurring in Brazil (BFG 2015). Psidium guajava L. was the only non-native species registered in our study (Sobral et al. 2016) while Ixora brevifolia Benth was the only species whose distribution is currently unknown (Di Maio 2016).

We registered 44 species endemic to the Atlantic Forest (31\%) (table 1). The Atlantic Forest is known by its high endemism (Myers et al. 2000; Ribeiro et al. 2011). However, the percentage of endemic species in this hotspot has slightly decreased from $50.2 \%$ in 2010 to $49.5 \%$ in 2015 (BFG 2015). Such reduction in the percentage of endemism can be justified by the increase in knowledge on the distribution of species and by the area of Atlantic Forest inserted in other vegetation domains, like the gallery forests in the Cerrado and the enclaves in the Caatinga (BFG 2015).

The most representative dispersal syndrome was zoochory, which was found in 108 species (77\%). The abiotic dispersal syndrome, on the other hand, was found in 32 species, which represent $23 \%$ of all sampled species (table 1). Our results are in line with the pattern found in the Atlantic Rainforest, in which, analogously to other tropical forests (Fleming 1979, Jordano 2000), zoochory is the predominant syndrome (Campassi 2006). Such pattern found in tropical forests is related to the absence of long seasonal periods (e.g., Morellato et al. 2000). In other words, rates of zoochorous dispersal decrease with increasing rainfall seasonality.

Forest fragmentation, fruit collection by humans, and long-term defaunation are the major factors responsible for the loss of interaction between dispersers and dispersed species (Fuentes 2000). Evidence that defaunation, especially of large frugivorous species, can lead to the disappearance of plant species with large seeds that significantly contribute to the maintenance of carbon stock in the Atlantic Forest has been reported (Bello et al. 2015). In that sense, it is worth noting that the studied remnant plays an important ecological role in the region, as it provides food to local fauna. However, performing a floristic survey and implementing management practices for conservation of animal species in the region are essential to maintain the more than $70 \%$ of angiosperm species therein.
A total 14 threatened species were recorded in our survey (table 2). The main threat to populations of Aspidosperma polyneuron Müll.Arg., Apuleia leiocarpa (Vogel) J.F. Macbr., Cedrela fissilis Vell., Dalbergia nigra (Vell.) Allemão ex Benth., Joannesia princeps Vell., Melanoxylon brauna Schott, and Zeyheria tuberculosa (Vell.) Bureau ex Verl. is related to predatory exploitation by the timber industry and rural producers for manufacture of laminate and solid wood furniture as well as of ornamental structures, all of which have been historically leading to population decline in those species (Biodiversitas 2008, Martinelli \& Moraes 2013, IUCN 2016). Trigynaea oblongifolia Schltdl. is considered rare (Martinelli \& Moraes 2013), and similarly to Brosimum glaziovii Taub. (IUCN 2016), the species has a restricted distribution, which is the major threatening factor to both species. Populations of T. oblongifolia are threatened by loss of habitat quality (Martinelli \& Moraes 2013). Euterpe edulis Mart. is threatened by its intense exploitation for palm heart harvest across the entire area where it occurs and by the consequent decline of its populations. Trattinnickia ferruginea Kuhlm. is a rare species (Giulietti et al. 2009), with populations restricted to well preserved Atlantic Forest remnants in Minas Gerais State; additionally, it is threatened by loss of its occurrence area and of habitat quality due to deforestation for charcoal production, pastures, and silviculture. Cariniana legalis (Mart.) Kuntze populations are declining not only due to intense exploitation for wood extraction, but also due to their occurrence in fertile lands widely used in agriculture. Virola bicuhyba (Schott) Warb. is endemic to ombrophilous and semideciduous Atlantic Forests and occurs preferentially in areas at advanced stages of regeneration or at climax (Martinelli \& Moraes 2013). V. bicuhyba has medicinal value and its wood has high economic importance. Its major threat is the reduction of its populations, which is mainly caused by selective extraction and habitat conversion (Martinelli \& Moraes 2013).

Besides the rare and threatened species recorded in our survey, the studied fragment has also other species classified under those categories, like Mascagnia velutina C.E. Anderson, which is rare (Giulietti et al. 2009) and is considered vulnerable due to high fragmentation of five among ten localities of its occurrence (Biodiversitas 2008), and Sinningia carangolensis Chautems, which is a rare species restricted to the region of Carangola municipality, Minas Gerais State, and is thus considered endangered 
Table 2. Rare and threatened species found in the present study at Santa Rita Farm, Faria Lemos municipality, Minas Gerais State, Brazil, according to the Red List of the IUCN (2016), Martinelli \& Moraes (2013), and Biodiversitas (2008).

\begin{tabular}{|c|c|c|c|c|}
\hline Family & Species & IUCN (2016) & $\begin{array}{c}\text { Martinelli \& } \\
\text { Moraes(2013) }\end{array}$ & Biodiversitas (2008) \\
\hline Apocynaceae & Aspidosperma polyneuron & $\begin{array}{l}\text { Endangered } \\
\text { Alacd }+2 \mathrm{~cd}\end{array}$ & - & - \\
\hline Annonaceae & Trigynaea oblongifolia & - & Endangered B1ab(iii) & - \\
\hline Arecaceae & Euterpe edulis & - & Vulnerable A1acd & Endangered A4cd \\
\hline Bignoniaceae & Zeyheria tuberculosa & Vulnerable A1cd & - & - \\
\hline Burseraceae & Trattinnickia ferruginea & - & $\begin{array}{c}\text { Endangered } \\
\text { B1ab(ii,iii)+2ab(ii,iii) }\end{array}$ & $\begin{array}{l}\text { Critically endangered } \\
\text { B1ab(ii,iii) + C2(ii) }\end{array}$ \\
\hline Euphorbiaceae & Joannesia princeps & Vulnerable A1cd & - & - \\
\hline \multirow[t]{3}{*}{ Fabaceae } & Apuleia leiocarpa & - & Vulnerable A2d & - \\
\hline & Dalbergia nigra & Vulnerable A1cd & Vulnerable A4bcd & $\begin{array}{l}\text { Vulnerable A2cd + } \\
\text { B2ab(iii) }\end{array}$ \\
\hline & Melanoxylon brauna & - & Vulnerable D2 & $\begin{array}{l}\text { Vulnerable A2cd }+ \\
\text { C2a(i) }\end{array}$ \\
\hline Lecythidaceae & Cariniana legalis & Vulnerable A1ac & Endangered A2cd & - \\
\hline Meliaceae & Cedrela fissilis & $\begin{array}{l}\text { Endangered } \\
\text { Alacd }+2 \mathrm{~cd}\end{array}$ & Vulnerable A2cd & - \\
\hline Moraceae & Brosimum glaziovii & $\begin{array}{l}\text { Endangered } \\
\mathrm{B} 1+2 \mathrm{bc}\end{array}$ & - & - \\
\hline Myristicaceae & Virola bicuhyba & - & Endangered A4ac & - \\
\hline Urticaceae & Coussapoa curranii & Vulnerable A1c & - & - \\
\hline
\end{tabular}

(Martinelli \& Moraes 2013, Biodiversitas 2008). Anthurium santaritensis Nadruz \& Croat is not under any threatened status in any of the analyzed lists, although according to the SpeciesLink platform (http:// splink.cria.org.br/) it occurs only at the Santa Rita Farm fragment (locality of the typus) and at Alegre and Vila Velha municipalities, Espírito Santo State. The species Dichorisandra leonii Aona \& M.C.E. Amaral, Dichorisandra rupicola Aona \& M.C.E. Amaral, Myrcia concisa Sobral \& Leoni, and Calyptranthes carangola Sobral \& Leoni were recently described (Sobral \& Leoni 2010; Aona-Pinheiro \& Amaral 2012; Sobral et al. 2016) based on material collected at Santa Rita Farm and its surroundings.

The studied remnant has important attributes that indicate its preservation degree as well as the importance of its conservation. Its high degree of preservation is demonstrated by the high values of richness, diversity, and evenness; by the high proportion of zoochorous species, which provide food to local fauna and thus contribute to the maintenance of ecological interactions; and by the presence of populations of several threatened species, two of which (Virola bicuhyba and Trattinnickia ferruginea) occur in mature forests (Martinelli \& Moraes 2013). The presence of the rare species Trigynaea oblongifolia and Trattinnickia ferruginea reiterates and subsidizes the importance of conserving the studied fragment, which is located in a key area for biodiversity conservation. Furthermore, our results reinforce the evidence found by Rezende et al. (2015), according to which areas located at the $0-1,000 \mathrm{~m}$ altitudinal range in the Seasonal Semideciduous Atlantic Forest deserve attention in terms of the need for conservation measures, due to their high species richness and high number of endemic and threatened species.

\section{Acknowledgements}

The authors are thankful to the owners of Santa Rita Farm, for allowing the performance of the study, and to Mr. Ademar and Mr. Ricardo, for their assistance in data collection in the field and identification of botanical specimens. 


\section{Literature cited}

Aona-Pinheiro, L.Y.S. \& Amaral, M.C.E. 2012. Four new species of Dichorisandra J.C. Mikan (Commelinaceae) from Southeast Brazil. Phytotaxa 48: 7-22.

APG III. 2009. An update of the Angiosperm Phylogeny Group classification for the orders and families of flowering plants: APG III. Botanical Journal of the Linnean Society 161: 105-121.

Aquino, A. \& Barbosa, L.M. 2009. Classes sucessionais e síndromes de dispersão de espécies arbóreas e arbustivas existentes em vegetação ciliar remanescente (Conchal, $\mathrm{SP}$ ), como subsídio para avaliar o potencial do fragmento como fonte de propágulos para enriquecimento de áreas revegetadas no rio Mogi-Guaçu, SP. Revista Árvore 33: 349-358.

Ayres, J.M., Fonseca, G.A.B., Rylands, A.B., Queiroz, H.L., Pinto, L.P., Masterson, D. \& Cavalcanti, R.B. 2005. Os corredores ecológicos das florestas tropicais do Brasil. Sociedade Civil Mamirauá, Belém.

Bello, C., Galetti, M., Pizo, M.A., Magnago L. F.S., Rocha, M. F., Lima, R.A.F., Peres, C.A., Ovaskainen, O. \& Jordano, P. 2015. Defaunation affects carbon storage in tropical forests. Science Advances 1: 1-11.

BFG. 2015. Growing knowledge: an overview of Seed Plant diversity in Brazil. Rodriguésia 66: 1085-1113.

Biodiversitas. 2008. Revisão das Listas das Espécies da Flora e da Fauna Ameaçadas de Extinção do Estado de Minas Gerais. Available in http://www.biodiversitas. org.br/listas-mg/consulta.asp (access in 24-IV-2016).

Braga, A.J.T., Lima e Borges, E.E. \& Martins, S.V. 2011. Florística e estrutura da comunidade arbórea de uma Floresta Estacional Semidecidual secundária em Viçosa, MG. Revista Árvore, Viçosa-MG. 35: 493-503.

Campassi, F. 2006. Padrões geográficos das síndromes de dispersão e características dos frutos de espécies arbustivo-arbóreas em comunidades vegetais na mata atlântica. Dissertação de Mestrado, Universidade de São Paulo, Piracicaba.

Catharino, E.L.M., Bernacci, L.C., Franco, G.A.D.C., Durigan, G. \& Metzger, J.P. 2006. Aspectos da composição e diversidade do componente arbóreo das florestas da Reserva Florestal do Morro Grande, Cotia, SP. Biota Neotropica 6.

Coelho, S., Cardoso-Leite, E. \& Castello, A.C.D. 2016. Composição florística e caracterização sucessional como subsídio para conservação e manejo do PNMCBio, Sorocaba - SP. Ciência Florestal 26: 331-344.

Di Maio, F.R. 2016. Ixora In: Lista de Espécies da Flora do Brasil. Jardim Botânico do Rio de Janeiro. Available in http://floradobrasil.jbrj.gov.br/jabot/floradobrasil/ FB14078 (access in 28-IV-2016).

Dias-Neto, O.C., Schiavini, I., Lopes, S.F., Vale, V.S., Gusson, A.E. \& Oliveira, A.P. 2009. Estrutura fitossociológica e grupos ecológicos em fragmento de Floresta Estacional Semidecidual, Uberaba, Minas Gerais, Brasil. Rodriguésia 60: 1087-1100.
Fleming, T.H. 1979. Do tropical frugivores completed for food? Annales Zoologici Fennici 19: 1157-1172.

Flora do Brasil 2020 (em construção). 2016. Jardim Botânico do Rio de Janeiro. Available in http:// floradobrasil.jbrj.gov.br/ (access in 28-IV-2016).

Fuentes, M. 2000. Frugivory, seed dispersal and plant community ecology. Trends in Ecology and Evolution 15: 487-488

Giulietti, A.M., Rapini, A., Andrade, M.J.G., Queiroz, L.P. \& Silva, J.M.C. 2009. Plantas raras do Brasil. Conservação Internacional, Belo Horizonte.

IUCN. 2016. Lista Vermelha de Espécies Ameaçadas de Extinção da União Internacional para a Conservação da Natureza. 2013. Available in http://www.iucnredlist.org (access in 20-IV-2016).

Jordano, P. 2000. Fruits and frugivory. In: M. Fenner (ed.). Seeds: The Ecology of Regeneration in Plant Communities. 2 ed. CAB International, Wallingford, pp. 125-165.

Kinoshita, L.S., Torres, R.B., Forni-Martins, E.R. \& Spinelli, T. 2006. Composição florística e síndromes de polinização e de dispersão da mata do Sítio São Francisco, Campinas, SP. Brasil. Acta Botanica Brasilica 20: 313-327.

Langhammer, P.F., Bakarr, M.I., Bennun, L.A., Brooks, T.M., Clay, R.P., Darwall, W., Silva, N., Edgar, G.J., Eken, G., Fishpool, L.D.C., Fonseca, G.A.B., Foster, M.N., Knox, D.H., Matiku, P., Radford, E.A., Rodrigues, A.S.L., Salaman, P., Sechrest, W. \& Tordoff, A.W. 2007. Identification and gap analysis of key biodiversity areas: Targets for comprehensive protected area systems. IUCN Best Practice Protected Areas Guidelines Series No 15, IUCN: Gland, Switzerland.

Leite, E.C. \& Rodrigues, R.R. 2008. Fitossociologia e caracterização sucessional de um fragmento de Floresta Estacional no sudeste do Brasil. Revista Árvore 32: 583-595.

Marangon, L.C., Soares, J.J., Feliciano, A.L.P. \& Silva Brandão, C.F. L. Estrutura fitossociológica e classificação sucessional do componente arbóreo de um fragmento de Floresta Estacional Semidecidual, no município de Viçosa, Minas Gerais. Cerne 13: 208-221.

Martinelli, G. \& Moraes, M.D. 2013. Livro Vermelho da Flora do Brasil. Andrea Jakobsson: Instituto de Pesquisas Jardim Botânico do Rio de Janeiro, Rio de Janeiro.

Meira-Neto, J.A.A. \& Martins, F.R. 2000. Estrutura da Mata da Silvicultura, uma Floresta Estacional Montana no município de Viçosa, MG. Revista Árvore 24: $151-160$.

Melo, A.S. 2008. O que ganhamos ‘confundindo' riqueza de espécies e equabilidade em um índice de diversidade? Biota Neotropica 8. 
Morellato, L.P.C., Talora, D.C., Takahasi, A., Bencke, C.C., Romera, E.C.\& Zipparro, V.B. 2000. Phenology of Atlantic Rain Forest Trees: A Comparative Study. Biotropica 32: 811-823.

Moro, M.F., Martins, F.R. 2011. Métodos de Levantamento do Componente Arbóreo-Arbustivo. In: J.M. Felfili., P.V. Eisenlohr., M.M.R.F. Melo., L.A. Andrade., J.A.A. Meira-Neto. (eds.). Fitossociologia no Brasil: métodos e estudos de casos. Editora UFV, Viçosa, pp.174-212.

Myers, N., Mittermeier, R.A., Mittermeier, C.G., Fonseca, G.A.B. \& Kent, J. 2000. Biodiversity hotspots for conservation priorities. Nature, 403: 853-858.

Oliveira-Filho, A.T. 2006. Catálogo das Árvores Nativas de Minas Gerais - Mapeamento e Inventário da Flora Nativa e dos Reflorestamentos de Minas Gerais. Editora UFLA, Lavras.

Oliveira-Filho, A.T. \& Fontes, M.A.L. 2000. Patterns of floristic differentiation among Atlantic Forests in southeastern Brazil and the influence of climate. Biotropica 32: 793-810.

Peel, M.C., Finlayson, B.L. \& Mcmahon, T.A. 2007. Updated world map of the Köppen Geiger climate classification. Hydrology and Earth System Sciences Discussions 11: 1633-1644.

Prado-Júnior, J.A., Lopes, S.F., Schiavini, I., Vale, V.S., Oliveira, A.P., Gusson, A.E., Dias-Neto, O.C. \& Stein, M. 2012. Fitossociologia, caracterização sucessional e síndromes de dispersão da comunidade arbórea de remanescente urbano de Floresta Estacional Semidecidual em Monte Carmelo, Minas Gerais. Rodriguésia 63: 489-499.

Ramos, V.S., Durigan, G., Franco, G.A.D.C., Siqueira, M.F. \& Rodrigues, R.R. 2008. Árvores da Floresta Estacional Semidecidual - Guia de Identificação de espécies. São Paulo: Editora da Universidade de São Paulo: Biota/Fapesp, São Paulo.

Rezende, V.L., Miranda, P.L.S., Meyer, L., Moreira, C.V., Linhares, M.F., Oliveira-Filho, A.T. \& Eisenlohr, P.V. 2015 Tree species composition and richness along altitudinal gradients as a tool for conservation decisions: the case of Atlantic Semideciduous Forest. Biodiversity and Conservation 24: 2255-2272.

Ribeiro, M.C., Metzger, J.P., Martensen, A.C., Ponzoni, F.J. \& Hirota, M.M. 2009. The Brazilian Atlantic Forest: How much is left, and how is the remaining forest distributed? Implications for conservation. Biological Conservation 142: 1141-1153.
Ribeiro, M.C., Martensen, A.C., Metzer, J.P., Tabarelli, M., Scarano, F. \& Fortin, M.-J. 2011. The Brazilian Atlantic Forest: a shrinking biodiversity hotspot. In: F.E. Zachos. \& J.C. Habel (eds.). Biodiversity hotspots: distribution and protection of conservation priority areas. Springer, Heidelberg/Dordrecht/London/New York, pp. 405-434.

Santos, M.L., Meira-Neto, J.A.A., Silva, A.F., Martins, S.V. \& Campos, E.P. 2013. Estrutura fitossociológica e raridade em um trecho de Floresta Estacional Semidecidual primária na zona da mata de Minas Gerais. Global Science and Technology 6: 101-117.

Silva, N.R.S., Martins, S.V., Meira-Neto, J.A.A. \& Souza, A.L. 2004. Composição florística e estrutura de uma Floresta Estacional Semidecidual Montana em Viçosa, MG. Revista Árvore 28: 397-405.

Sobral, M. \& Leoni, L.S. 2010. Myrcia concisa (Myrtaceae), a New Species from Minas Gerais, Brazil. Novon 20: 345-347.

Sobral, M., Mazine, F.F., Leoni, L., Souza, M.C. \& Melo, E.A.D. 2016. Five new southeastern Brazilian Myrtaceae. Phytotaxa 253: 57-70.

Sobral, M., Proença, C., Souza, M., Mazine, F. \& Lucas, E. 2016. Myrtaceae. In: Lista de Espécies da Flora do Brasil. Jardim Botânico do Rio de Janeiro. Available in http://floradobrasil.jbrj.gov.br/jabot/floradobrasil/ FB24034 (access in 18-IV-2016).

Stehmann, J.R., Forzza, R.C., Sobral, M. \& Kamino, L.H.Y. 2009. Gimnospermas e angiospermas. In: J.R. Stehmann, R.C. Forzza, M. Sobral \& L.H.Y. Kamino (eds.). Plantas da Floresta Atlântica. Jardim Botânico do Rio de Janeiro, Rio de Janeiro, pp. 27-37.

van der Pijl, L. 1982. Principles of dispersal in higher plants. 2 ed. Springer-Verlag, Berlin.

Yamamoto, L.F., Kinoshita, L.S. \& Martins, F. R. 2007. Síndromes de polinização e de dispersão em fragmentos da Floresta Estacional Semidecídua Montana, SP, Brasil. Acta Botanica Brasilica 21: 553-573.

Zachos, F. \& Habel, J. 2011. Biodiversity hotspots: distribution and protection of conservation priority areas. Springer. Heidelberg/Dordrecht/ London/New York. 